\section{Fatores associados à idade da primeira relação sexual em jovens: estudo de base populacional}

\author{
Factors associated with age at first intercourse: \\ a population-based study
}

\author{
1 Universidade Católica de \\ Pelotas, Pelotas, Brasil. \\ Correspondência \\ K. Jansen \\ Universidade Católica de \\ Pelotas. \\ Rua Santos Dumont 257, \\ apto. 301, Pelotas, RS \\ 96020-380, Brasil. \\ jansen@ucpel.tche.br
}

\begin{abstract}
First sexual intercourse is considered an important event in young people's lives and has occurred at an increasingly early age. The aim of this study was to identify factors associated with early age at first intercourse in individuals 18 to 24 years of age in Pelotas, Rio Grande do Sul State, Brazil. This was a population-based cross-sectional study in a representative sample of 1,621 young people from August 2007 to December 2008. Subjects answered a questionnaire on health behaviors, including items related to their sexual lives. Cox regression was used to assess the association between early age at sexual initiation. After multivariate analysis, variables that are directly related to early sexual initiation were: male gender, low socioeconomic status, low schooling, divorced parents, living with a partner, not practicing a religion, smoking, drug use in the previous three months, and non-use of condoms during last intercourse. Considering the current social context, the study highlights the need for adequate sexual orientation with a preventive approach.
\end{abstract}

Sexual Behavior; Safe Sex; Adolescent; Street Drugs
Tairana Dias de Oliveira Hugo ${ }^{1}$

Vanessa Teixeira Maier 1

Karen Jansen 1

Cristine Eliane Gomes Rodrigues 1

Ana Laura Sicca Cruzeiro 1

Liliane da Costa Ores 1

Ricardo Tavares Pinheiro 1

Ricardo Silva 1

Luciano Dias de Mattos Souza 1

\section{Introdução}

A primeira relação sexual, considerada um marco na vida dos jovens, tem iniciado cada vez mais precocemente. No contexto brasileiro, a idade média da primeira relação sexual é de 14 anos para o sexo masculino e 15 para o feminino ${ }^{1}$.

As mulheres priorizam o sentimento de "entrega e amor" na primeira relação sexual, ao mesmo tempo em que existe o desejo de se descobrir, impõe-se a necessidade de se "preservar". Em contrapartida, a experiência sexual masculina é vista como um ganho, sustentando o poder da masculinidade 2. Estudo revela que jovens tendem a não usar preservativo no início de sua vida sexual e definem esta relação como casual. Os principais motivos alegados para a sua não utilização de modo consistente são: não gostar de usá-los, confiar no parceiro e a imprevisibilidade das relações sexuais ${ }^{3}$.

Além disso, há uma relação entre o uso de álcool e outras substâncias psicoativas com comportamentos sexuais de risco na adolescência 4 . Ademais, investigações indicam que a iniciação sexual precoce está associada a um maior risco de contaminação por doenças sexualmente transmissíveis (DST) e gravidez na adolescência 5,6,7. A literatura científica sugere que os fatores associados à iniciação sexual dependem de aspectos culturais. O uso de substâncias apresenta associação significativa com iniciação sexual precoce em países europeus e nos Estados Unidos, 
contudo, tal associação é mais forte na Europa ${ }^{8}$. $\mathrm{O}$ mesmo estudo indica que a escolaridade do jovem também apresenta relação inversamente proporcional com a idade da primeira relação sexual 8. Já em pesquisa realizada em países africanos, a escolaridade e o uso de álcool apresentaram relação significativa com a iniciação sexual precoce apenas entre as meninas, enquanto o consumo de tabaco esteve associado à iniciação sexual precoce somente entre a população masculina ${ }^{9}$.

A elaboração de estratégias educacionais que visem à minimização de desfechos negativos em saúde relacionados à iniciação sexual precoce depende da identificação de características dos jovens que iniciam sua vida sexual mais cedo. Portanto, o objetivo deste estudo foi descrever os fatores associados à idade da primeira relação sexual, com uma amostra populacional de jovens de 18 a 24 anos, residentes na zona urbana da cidade de Pelotas, Rio Grande do Sul, Brasil.

\section{Métodos}

Trata-se de um estudo quantitativo transversal de base populacional, em uma amostra representativa de jovens de 18 a 24 anos de idade, residentes da zona urbana da cidade de Pelotas, que ocorreu entre agosto de 2007 a dezembro de 2008.

A presente investigação se insere em um estudo que visou à avaliação de comportamentos de saúdena população dejovens, com foco principal na depressão. $\mathrm{O}$ universo em estudo correspondeu à população de 39.667 jovens em 448 setores censitários, segundo o Instituto Brasileiro de Geografia e Estatística (IBGE; http://www.ibge. gov.br, acessado em Mai/2008), na faixa etária compreendida entre os 18 a 24 anos. Para o cálculo da amostra, usando-se o programa Epi Info 6.04d (Centers for Disease Control and Prevention, Atlanta, Estados Unidos), foi utilizada a estimativa de depressão de $10 \%$, intervalo de $95 \%$ de confiança e erro amostral de $2 \%$. Foram ainda acrescidos $30 \%$ para controle de fatores de confusão, perdas e recusas, e a amostra necessária foi de 1.100 indivíduos. Realizou-se os cálculos de tamanho amostral para aferição de associação entre a idade da primeira relação sexual e as variáveis independentes em estudo. Apenas em relação à associação entre o número de parceiros sexuais no último ano e o desfecho em questão seria necessária a participação de um número maior $(\mathrm{n}=1.571)$ do que o anteriormente descrito.

A seleção amostral foi realizada por conglomerados. Foram sorteados 89 setores para a identificação dos jovens que participaram da in- vestigação. A escolha dos domicílios nos setores sorteados foi realizada segundo uma amostragem sistemática, sendo o primeiro domicílio a residência da esquina preestabelecida pelo IBGE como início do setor, e o intervalo de seleção foi determinado por um pulo de dois domicílios entre os sorteados. Foram excluídos da amostra aqueles jovens identificados pela equipe de pesquisa que residiam na zona não urbana de Pelotas ou que apresentaram dificuldade em compreender e/ou responder ao questionário.

Para a coleta das informações utilizadas na presente pesquisa foi utilizada uma entrevista estruturada que contém questões referentes à saúde e comportamento dos jovens. Nessa entrevista, o aplicador seguiu um questionário que aborda itens como: idade, uso de drogas, sexualidade e outros comportamentos de risco à saúde. Em um instrumento de autorrelato o jovem forneceu informações sobre comportamento sexual e abuso de substâncias.

Para avaliação do abuso de substâncias foi utilizado o teste de triagem do envolvimento com álcool, drogas ilícitas e outras substâncias (ASSIST), um instrumento desenvolvido por pesquisadores de vários países sob a coordenação da Organização Mundial da Saúde (OMS) e traduzido para vários idiomas, já tendo sido testado quanto à sua confiabilidade e factibilidade 10 .

A sintomatologia depressiva foi avaliada utilizando-se o Inventário Beck de Depressão (BDI). Esse possui 21 itens nos quais constam frases que avaliam a intensidade de sintomas depressivos, de forma breve e em escala Likert de 0 a 3 pontos. Portanto, esse instrumento oferece uma pontuação total que pode variar de 0 a 63 . Conforme estudo de validação 11, o ponto de corte utilizado para indicativo de depressão foi de 20 pontos ou mais.

Duas questões referentes ao comportamento sexual de risco foram consideradas para o desfecho: a primeira, “Tu já tiveste relações sexuais?", tinha como opções de respostas não/sim; a segunda (aferida apenas para aqueles jovens que responderam sim anteriormente), "Que idade tinha na tua primeira relação sexual?”, foi analisada como contínua (em anos). Para garantir a qualidade das informações utilizadas nesta pesquisa, o questionário autoaplicado foi usado para assegurar o sigilo dos dados e encorajar os jovens a serem o mais fidedignos com os fatos ao longo de suas vidas. A equipe de entrevistadores recebeu treinamento para homogeneizar os procedimentos de abordagem nas residências, assinatura do termo de consentimento após esclarecimentos sobre o estudo para os jovens identificados, e aplicação dos instrumentos e entrevista. O projeto foi aprovado pelo Comitê 
de Ética em Pesquisa da Universidade Católica de Pelotas (UCPel), que obteve aprovação sob o protocolo 2006/96.

O programa Epi Info 6.04d foi utilizado para a dupla digitação dos dados e na checagem automática da amplitude e consistência. As análises estatísticas foram realizadas por meio dos programas Stata 9 (Stata Corp., College Station, Estados Unidos) e SPSS 13.0 (SPSS Inc., Chicago, Estados Unidos). Após a obtenção de frequência simples de todas as variáveis em estudo, ocorreu a análise bivariada. Nela, foi verificada a relação estatística entre a iniciação sexual e as variáveis independentes. Nessa análise estatística foi utilizada a análise de sobrevivência ${ }^{12}$. A regressão de Cox foi utilizada na análise multivariada e seguiu um modelo hierárquico, no qual cada bloco de variáveis de um determinado nível foi incluído e as variáveis com valor de $\mathrm{p}<0,20$ no teste de razões de verossimilhança permaneciam no modelo. Nesse tipo de modelo, as variáveis situadas em um nível hierárquico superior ao da variável em questão são consideradas como potenciais confundidores da relação entre essa variável e o desfecho em estudo, enquanto que as variáveis em níveis inferiores são consideradas como potenciais mediadores da associação. As variáveis selecionadas em um determinado nível permaneceram nos modelos subsequentes e foram consideradas como fatores associados com a iniciação sexual mesmo que, com a inclusão de variáveis hierarquicamente inferiores, tivessem perdido sua significância.

\section{Resultados}

A pesquisa identificou 1.823 jovens entre 18 a 24 anos de idade. Desses, 1.621 aceitaram participar do estudo e realizaram a entrevista de forma completa. Do total de participantes, 1.468 indivíduos já haviam tido sua primeira relação sexual. A média de idade da primeira relação foi de 15,7 anos (DP = 1,98) (Tabela 1).

Nessa amostra foram considerados 1.468 indivíduos, sendo que destes, $54 \%$ correspondem ao sexo feminino. Entre os jovens entrevistados, os que estudaram de 9 a 11 anos tiveram uma maior prevalência $(40,7 \%)$, sendo assim, estes não chegaram a concluir o Ensino Médio. Do total de participantes, 37,9\% têm os pais separados e 47,5\% pertencem à classe socioeconômica C. Com relação à prática religiosa, 30,5\% dos jovens relataram ser praticantes de alguma religião.

Dentre os entrevistados, um número significativo de $73,2 \%$ de jovens consumiram álcool nos últimos três meses, enquanto 13,9\% fizeram uso de drogas ilícitas e 31,7\% fumaram nos últimos três meses. O indicativo de depressão foi observado em $7,8 \%$ dos entrevistados. No que tange às variáveis relacionadas à sexualidade dos jovens, $83,1 \%$ não consumiram bebida alcoólica antes da última relação sexual. De acordo com a amostra, foi observado que $58 \%$ dos jovens entrevistados usaram camisinha na última relação sexual, a maioria manteve um parceiro fixo $(74,1 \%)$ e $66,1 \%$ tiveram um único parceiro no último ano (Tabela 1).

O risco de ter iniciação sexual em uma idade mais jovem foi $41 \%$ maior entre os homens quando comparados com as mulheres. Evidenciou-se uma relação inversamente proporcional entre nível socioeconômico e risco de iniciação sexual precoce, ou seja, quanto menor o nível socioeconômico do jovem maior o risco para iniciação sexual precoce.

Com relação à escolaridade, quanto menor ela for maior o risco para o início precoce da atividade sexual. Jovens que tinham até quatro anos de estudos apresentaram risco $41 \%$ acrescido de iniciação sexual precoce em comparação com aqueles com 12 anos ou mais de estudos. Ter pais separados influenciou na iniciação precoce do adolescente (HR: 1,23; IC95\%: 1,10-1,37), assim como a não prática da religião (HR: 1,18; IC95\%: 1,05-1,33). Adolescentes que fizeram uso de drogas e fumaram nos últimos três meses também mostraram risco significativamente acrescido para menor idade no início da prática sexual $(\mathrm{p}<0,05)$. Morar com companheiro atualmente também mostrou proporção significativamente acrescida em $20 \%$ de jovens que tiveram iniciação da vida sexual precoce. Não houve diferença estatisticamente significativa da idade da primeira relação sexual entre os grupos de jovens que apresentaram indicativo de depressão e aqueles que não o apresentaram (Tabela 2).

Mostrou-se relevante a diferença de proporções com relação ao uso da camisinha na última relação. Os jovens que não fizeram uso da camisinha na última relação apresentaram iniciação sexual cedo (HR: 1,17; IC95\%: 1,04-1,31). Ter bebido antes da última relação e o número de parceiros no último ano não mostraram associação estatisticamente significativa com o início precoce da atividade sexual (Tabela 2).

\section{Discussão}

Nesta amostra de 1.621 jovens, o sexo masculino, baixo nível socioeconômico, pouca escolaridade, ter pais separados, morar com companheiro(a), não praticar uma religião, uso de tabaco e drogas e o não uso da camisinha na 
Caracterização da amostra de jovens entre 18-24 anos da cidade de Pelotas, Rio Grande do Sul, Brasil, 2007/2008.

\begin{tabular}{|c|c|c|}
\hline Variáveis & $\mathrm{n}$ & $\%$ \\
\hline \multicolumn{3}{|l|}{ Sexo } \\
\hline Masculino & 675 & 46,0 \\
\hline Feminino & 793 & 54,0 \\
\hline \multicolumn{3}{|c|}{ Nível socioeconômico } \\
\hline$A+B$ & 554 & 37,7 \\
\hline C & 698 & 47,5 \\
\hline$D+E$ & 216 & 14,7 \\
\hline \multicolumn{3}{|c|}{ Escolaridade (anos) } \\
\hline $0-4$ & 103 & 7,0 \\
\hline $5-8$ & 335 & 22,9 \\
\hline $9-11$ & 595 & 40,7 \\
\hline 12 ou mais & 429 & 29,3 \\
\hline \multicolumn{3}{|c|}{ Tem pais separados } \\
\hline Não & 909 & 62,1 \\
\hline Sim & 554 & 37,9 \\
\hline \multicolumn{3}{|c|}{ Prática religiosa } \\
\hline Não & 1.016 & 69,5 \\
\hline Sim & 446 & 30,5 \\
\hline \multicolumn{3}{|c|}{ Bebeu álcool nos últimos três meses } \\
\hline Não & 430 & 26,8 \\
\hline Sim & 1.173 & 73,2 \\
\hline \multicolumn{3}{|c|}{ Fumou nos últimos três meses } \\
\hline Não & 1.095 & 68,3 \\
\hline Sim & 508 & 31,7 \\
\hline \multicolumn{3}{|c|}{ Drogas nos últimos três meses } \\
\hline Não & 1.236 & 86,1 \\
\hline Sim & 199 & 13,9 \\
\hline \multicolumn{3}{|c|}{ Indicativo de depressão } \\
\hline Sim & 126 & 7,8 \\
\hline Não & 1.492 & 92,2 \\
\hline \multicolumn{3}{|c|}{ Tu usaste camisinha na última relação sexual? } \\
\hline Não & 615 & 41,9 \\
\hline Sim & 849 & 58,0 \\
\hline \multicolumn{3}{|c|}{ Tu tomaste alguma bebida alcoólica antes da última } \\
\hline \multicolumn{3}{|c|}{ relação sexual? } \\
\hline Não & 1.216 & 82,8 \\
\hline Sim & 247 & 16,9 \\
\hline \multicolumn{3}{|c|}{ Tu tens parceiro(a) fixo? } \\
\hline Não & 377 & 25,9 \\
\hline Sim & 1.081 & 74,1 \\
\hline \multicolumn{3}{|c|}{ Número de parceiros(as) no último ano } \\
\hline 1 & 937 & 66,1 \\
\hline 2 ou 3 & 266 & 18,8 \\
\hline 4 ou mais & 215 & 15,2 \\
\hline
\end{tabular}


Modelo hierárquico da regressão de Cox para iniciação sexual precoce. Pelotas, Rio Grande do Sul, Brasil, 2007/2008.

\begin{tabular}{|c|c|c|c|c|}
\hline Variável & HR bruta (IC95\%) & Valor de $p$ & HR ajustada (IC95\%) & Valor de $p$ \\
\hline \multicolumn{5}{|l|}{ Primeiro nível } \\
\hline Sexo & & 0,000 & & 0,000 \\
\hline Masculino & $1,39(1,25-1,54)$ & & $1,41(1,27-1,56)$ & \\
\hline Feminino & Referência & & Referência & \\
\hline Nível socioeconômico & & 0,001 & & 0,000 \\
\hline$A$ ou $B$ & Referência & & Referência & \\
\hline C & $1,10(0,98-1,23)$ & 0,095 & $1,13(1,01-1,27)$ & 0,032 \\
\hline D ou $E$ & $1,35(1,15-1,59)$ & 0,000 & $1,39(1,19-1,64)$ & 0,000 \\
\hline \multicolumn{5}{|l|}{ Segundo nível } \\
\hline Escolaridade do adolescente (anos) & & 0,000 & & 0,001 \\
\hline $0-4$ & $1,68(1,35-2,09)$ & 0,000 & $1,41(1,11-1,79)$ & 0,006 \\
\hline $5-8$ & $1,61(1,39-1,86)$ & 0,000 & $1,39(1,17-1,65)$ & 0,000 \\
\hline $9-11$ & $1,23(1,09-1,40)$ & 0,001 & $1,20(1,04-1,37)$ & 0,010 \\
\hline 12 ou mais & Referência & & Referência & \\
\hline Tem pais separados & & 0,000 & & 0,000 \\
\hline Sim & $1,36(1,22-1,52)$ & & $1,23(1,10-1,37)$ & \\
\hline Não & Referência & & Referência & \\
\hline Mora com companheiro & & 0,000 & & 0,004 \\
\hline Sim & $1,25(1,12-1,40)$ & & $1,21(1,07-1,38)$ & \\
\hline Não & Referência & & Referência & \\
\hline Pratica religião & & 0,000 & & 0,005 \\
\hline Sim & Referência & & Referência & \\
\hline Não & $1,30(1,16-1,45)$ & & $1,18(1,05-1,33)$ & \\
\hline Uso de drogas nos últimos 3 meses & & 0,000 & & 0,016 \\
\hline Sim & $1,32(1,13-1,53)$ & & $1,22(1,04-1,43)$ & \\
\hline Não & Referência & & Referência & \\
\hline Bebeu nos últimos 3 meses & & 0,022 & & 0,200 \\
\hline Sim & $1,15(1,02-1,30)$ & & $1,09(0,96-1,24)$ & \\
\hline Não & Referência & & Referência & \\
\hline Fumou nos últimos 3 meses & & 0,000 & & 0,003 \\
\hline Sim & $1,35(1,21-1,50)$ & & $1,21(1,07-1,36)$ & \\
\hline Não & Referência & & Referência & \\
\hline Indicativo de depressão & & 0,686 & & \\
\hline Sim & $0,92(0,62-1,38)$ & & & \\
\hline Não & Referência & & & \\
\hline \multicolumn{5}{|l|}{ Terceiro nível } \\
\hline Uso de camisinha na última relação & & 0,000 & & 0,007 \\
\hline Sim & Referência & & Referência & \\
\hline Não & $1,22(1,10-1,35)$ & & $1,17(1,04-1,31)$ & \\
\hline Bebeu antes da última relação & & 0,082 & & 0,935 \\
\hline Sim & $1,13(0,98-1,30)$ & & $1,01(0,86-1,17)$ & \\
\hline Não & Referência & & Referência & \\
\hline Número de parceiros(as) no último ano & & 0,000 & & 0,000 \\
\hline Nenhum & Referência & & Referência & \\
\hline Até 1 & $0,92(0,69-1,22)$ & 0,557 & $0,91(0,67-1,23)$ & 0,538 \\
\hline 2 ou 3 & $1,06(0,78-1,44)$ & 0,701 & $1,08(0,79-1,49)$ & 0,619 \\
\hline 4 ou mais & $1,44(1,06-1,96)$ & 0,021 & $1,33(0,96-1,83)$ & 0,088 \\
\hline
\end{tabular}

HR: hazard ratio; IC95\%: intervalo de 95\% de confiança. 
última relação se mostraram associados à iniciação sexual precoce.

Assim como aponta a literatura 7,13,14, o nosso estudo também mostra que o sexo masculino inicia sua vida sexual mais cedo quando comparado com o sexo feminino. As diferentes influências sociais e culturais de gênero relativas à prática sexual que acometem homens e mulheres podem explicar essa informação. Enquanto os homens têm sua iniciação sexual exigida como uma etapa simbólica de passagem à vida adulta, de forma oposta as mulheres ainda são pressionadas para abstinência antes do matrimônio.

Nível socioeconômico e escolaridade baixa são fatores que parte da literatura indica como associados ao início da atividade sexual precoce, assemelhando-se aos resultados do presente estudo 8,15,16. Ter baixa renda familiar e pouca escolaridade pode ter uma forte influência para o começo de uma vida sexual precoce devido à antecipação de algumas etapas evolutivas. A vulnerabilidade social entre os jovens impõe a necessidade de trabalhar mais cedo, assumir maiores responsabilidades com o próprio sustento e dos que com ele moram, antecipando em anos algumas condutas, inclusive sexual 17. Tal explicação parece ser a mais congruente com o contexto estudado. Contudo, a relação da iniciação sexual precoce com tais condições sociais desfavoráveis ainda é inconsistente 18,19 .

Da mesma forma, encontra-se referencial semelhante aos achados deste estudo com relação à associação da idade precoce de iniciação sexual e não praticar uma religião $3,13,18$, assim como investigações que não evidenciaram tal relação 15,19. Pode-se entender a religião como outro fator cultural que gera impacto no comportamento sexual, dependendo da representatividade social e do contexto estudado.

Apesar deste estudo não ter encontrado associação significativa entre o uso de álcool e o desfecho, assim como em outros trabalhos, o uso de tabaco e drogas ilícitas esteve associado à iniciação sexual precoce ${ }^{8,13}$. Acredita-se que a pergunta utilizada para aferição do consumo de álcool (alguma vez nos últimos três meses) possui grande sensibilidade, dificultando a diferenciação entre grupos de usuários e não usuários.
O indicativo de depressão não esteve associado à iniciação sexual precoce na amostra investigada. A associação entre essas variáveis se apresentou significativa em estudo realizado em Hong Kong, China 20. Contudo, assim como a presente investigação, outras pesquisas têm sinalizado a inconsistência dessa associação apontando a influência de fatores socioculturais e explicitando a necessidade de estudos longitudinais sobre a relação de sintomas depressivos e a iniciação sexual 21,22.

Com relação aos comportamentos sexuais de risco, estudos nacionais e internacionais indicam que a iniciação sexual precoce se relaciona com um padrão de comportamento sexual de risco 1,23 . Os hábitos sexuais começam no início da vida sexual e perduram ao longo da vida, por isto a importância do comportamento seguro desde a adolescência 24 . Os achados deste estudo sugerem que esse padrão pode persistir por toda a vida do jovem com relação ao uso de preservativos. Contudo, de forma discrepante da literatura científica 23,25 , este estudo aponta que a iniciação sexual precoce não está associada ao comportamento sexual de risco no que se refere à ingestão de bebida alcoólica antes da última relação e ao número de parceiros no último ano. Esse último resultado deve ser interpretado com cautela em função do número de participantes que haviam iniciado sua vida sexual inquiridos na presente investigação.

É marcadamente sabido que um início sexual precoce acarreta não só mais parceiros ao longo da vida, mas também chances maiores de doenças sexuais, comportamento antissocial e gestações indesejadas, e está intimamente ligado às bases familiares e experiências de amigos 26,27. A educação sexual formal pode auxiliar a minimizar os riscos da iniciação sexual precoce 28 . Considerando que o início da vida sexual dos adolescentes ocorre cada vez mais cedo, as participações da família, da escola, de campanhas voltadas à prevenção de DST fazem-se necessárias e devem dirigir esforços para orientar os jovens com relação às DST e gestações indesejadas. Essas iniciativas ajudarão na promoção da saúde e bem-estar dos jovens. 


\section{Resumo}

A primeira relação sexual é considerada um evento importante na vida dos jovens e tem iniciado cada vez mais cedo. O objetivo do presente estudo foi descrever os fatores relacionados à idade precoce da primeira relação sexual, de jovens de 18 a 24 anos na cidade de Pelotas, Rio Grande do Sul, Brasil. Este é um estudo transversal de base populacional, em uma amostra representativa de 1.621 jovens que ocorreu entre agosto de 2007 e dezembro de 2008. Foram aplicados questionários sobre comportamentos de saúde que continham itens relativos à sexualidade. A regressão de Cox foi utilizada para aferir a associação com a idade precoce de início da vida sexual. Após a análise multivariada, as variáveis diretamente relacionadas com a iniciação sexual precoce foram: sexo masculino, baixo nível socioeconômico, baixa escolaridade, ter pais separados, morar com companheiro(a), não praticar uma religião, uso de tabaco e drogas, e o não uso da camisinha na última relação. Considerando o contexto social atual, evidencia-se a necessidade de uma adequada orientação sexual com aspecto preventivo.

Comportamento Sexual; Sexo Seguro; Adolescente; Drogas Ilícitas

\section{Colaboradores}

K. Jansen, T. D. O. Hugo, C. E. G. Rodrigues, A. L. S. Cruzeiro e L. C. Ores contribuíram com a interpretação dos dados, redação do artigo e aprovação da versão a ser publicada. R. Silva contribuiu com a concepção e delineamento do estudo, redação do artigo e aprovação da versão a ser publicada. R. T. Pinheiro participou da concepção e delineamento do estudo, revisão crítica e aprovação da versão a ser publicada. L. D. M. Souza contribuiu com a análise e interpretação dos dados, redação do artigo e aprovação da versão a ser publicada. V. T. Maier participou da redação do artigo, análise e interpretação dos dados, revisou e aprovou a versão final do artigo.

\section{Referências}

1. Borges ALV, Schor N. Início da vida sexual na adolescência e relações de gênero: um estudo transversal em São Paulo, Brasil. Cad Saúde Pública 2005; 21:499-507.

2. Borges ALV, Nakamura E. Normas sociais de iniciação sexual entre adolescentes e relações de gênero. Rev Latinoam Enferm 2009; 17:94-100.

3. Paiva V, Calazans G, Venturi G, Dias R. Idade e uso de preservativo na iniciação sexual de adolescentes. Rev Saúde Pública 2008; 42:45-53.

4. Cruzeiro ALS, Souza LDM, Silva RA, Pinheiro RT, Rocha CLA, Horta BL. Comportamento sexual de risco: fatores associados ao número de parceiros sexuais e ao uso de preservativo em adolescentes. Ciênc Saúde Coletiva 2010; 15:1149-58.
5. Carret ML, Fassa AG, Silveira DS, Bertoldi AD, Hallal PC. Sexually transmitted diseases symptoms in adults: prevalence and risk factors. Rev Saúde Pública 2004; 38:76-84.

6. Sandfort TGM, Orr M, Hirsch JS, Santelli J. Longterm health correlates of timing of sexual debut: results from a National US Study. Am J Public Health 2008; 98:155-61.

7. Ma Q, Ono-Kihara M, Cong L, Xu G, Pan X, Zamani S, et al. Early initiation of sexual activity: a risk factor for sexually transmitted diseases, HIV infection, and unwanted pregnancy among university students in China. BMC Public Health 2009; 9:111-9. 
8. Madkour AS, Farhat T, Halpern CT, Godeau E, Gabhainn SN. Early adolescent sexual initiation as a problem behavior: a comparative study of five nations. J Adolesc Health 2010; 47:389-98.

9. Peltzer K. Early sexual debut and associated factors among in-school adolescents in eight African countries. Acta Paediatr 2010; 99:1242-7.

10. Henrique IFS, de Micheli D, Lacerda RB, Lacerda LA, Formigoni MLOS. Validação da versão brasileira do teste de triagem do envolvimento com álcool, cigarro e outras substâncias (ASSIST). Rev Assoc Med Bras 2004; 50:199-206.

11. Cunha JA. Manual da versão em português das escalas Beck. São Paulo: Casa do Psicólogo; 2001.

12. Thomas L, Stepanova M. Survival analysis methods of personal loan data. Oper Res 2002; 2:277-89.

13. Cruzeiro ALS, Souza LDM, Silva RA, Horta BL, Muenzer RM, Faria AD, et al. Iniciação sexual entre adolescentes de Pelotas, Rio Grande do Sul. Rev Bras Crescimento Desenvol Hum 2008; 18:116-25.

14. Rebello LEFS, Gomes R. Iniciação sexual, masculinidade e saúde: narrativas de homens jovens universitários. Ciênc Saúde Coletiva 2009; 14:653-60.

15. Fatusi AO, Blum RW. Predictors of early sexual initiation among a nationally representative sample of Nigerian adolescents. BMC Public Health 2008; 25:136.

16. Tavares CM, Schor N, França Jr. I, Diniz SG. Factors associated with sexual initiation and condom use among adolescents on Santiago Island, Cape Verde, West Africa. Cad Saúde Pública 2009; 25:1969-80.

17. Gonçalves HB, Gigante DP, Minten DP, Horta GC, Horta BL, Victora CG, et al. Determinantes sociais da iniciação sexual precoce na coorte de nascimentos de 1982 a 2004-5, Pelotas, RS. Rev Saúde Pública 2008; 42:34-41.

18. Sinha JW, Cnaan RA, Gelles RJ. Adolescent risk behaviors and religion: findings from a national study. J Adolesc 2007; 30:231-49.

19. Penfold SC, van Teijlingen ER, Tucker JS. Factors associated with self-reported first sexual intercourse in Scottish adolescents. BMC Res Notes 2009; 19:42-8.
20. Lam TH, Stewart SM, Leung GM, Lee PW, Wong JP, Ho LM, et al. Depressive symptoms among Hong Kong adolescents: relation to atypical sexual feelings and behaviors, gender dissatisfaction, pubertal timing, and family and peer relationships. Arch Sex Behav 2004; 33:487-96.

21. Sabia JJ. Does early adolescent sex cause depressive symptoms? J Policy Anal Manage 2006; 25: 803-25.

22. Jamieson LK, Wade TJ. Early age of first sexual intercourse and depressive symptomatology among adolescents. J Sex Res 2010; 14:1-11.

23. Langille DB, Asbridge M, Flowerdew G, Allen M. Associations of sexual risk-taking with having intercourse before 15 years in adolescent females in Cape Breton, Nova Scotia, Canada. Sex Health 2010; 7:199-204.

24. Stulhofer A, Bacak V, Ajdukovic D, Graham C. Understanding the association between condom use at first and most recent sexual intercourse: an assessment of normative, calculative, and habitual explanations. Soc Sci Med 2010; 70:2080-4.

25. Ohene SA, Ireland M, Blum RW. The clustering of risk behaviors among Caribbean youth. Matern Child Health J 2005; 9:91-100.

26. Boislard PMA, Poulin F. Individual, familial, friends-related and contextual predictors of early sexual intercourse. J Adolesc 2011; 34:289-300.

27. Villela WVD, Doreto DT. Sobre a experiência sexual dos jovens. Cad Saúde Pública 2006; 22:2467-72.

28. Mueller TE, Gavin LE, Kulkarni A. The association between sex education and youth's engagement in sexual intercourse, age at first intercourse, and birth control use at first sex. J Adolesc Health 2008; 42:89-96.

Recebido em 08/Ago/2010

Versão final reapresentada em 25/Mai/2011 Aprovado em 28/Jul/2011 